\title{
CUDA: Contradistinguisher for Unsupervised Domain Adaptation
}

\author{
Sourabh Balgi* and Ambedkar Dukkipati ${ }^{\dagger}$ \\ Department of Computer Science and Automation \\ Indian Institute of Science, Bengaluru, India \\ E-mail: *sourabhbalgi@iisc.ac.in, †ambedkar@iisc.ac.in
}

\begin{abstract}
Humans are very sophisticated in learning new information on a completely unknown domain because humans can contradistinguish, i.e., distinguish by contrasting qualities. We learn on a new unknown domain by jointly using unsupervised information directly from unknown domain and supervised information previously acquired knowledge from some other domain. Motivated by this supervised-unsupervised joint learning, we propose a simple model referred as Contradistinguisher (CTDR) for unsupervised domain adaptation whose objective is to jointly learn to contradistinguish on unlabeled target domain in a fully unsupervised manner along with prior knowledge acquired by supervised learning on an entirely different domain. Most recent works in domain adaptation rely on an indirect way of first aligning the source and target domain distributions and then learn a classifier on labeled source domain to classify target domain. This approach of indirect way of addressing the real task of unlabeled target domain classification has three main drawbacks. (i) The subtask of obtaining a perfect alignment of the domain in itself might be impossible due to large domain shift (e.g., language domains). (ii) The use of multiple classifiers to align the distributions, unnecessarily increases the complexity of the neural networks leading to over-fitting in many cases. (iii) Due to distribution alignment, the domain specific information is lost as the domains get morphed. In this work, we propose a simple and direct approach that does not require domain alignment. We jointly learn CTDR on both source and target distribution for unsupervised domain adaptation task using contradistinguish loss for the unlabeled target domain in conjunction with supervised loss for labeled source domain. Our experiments show that avoiding domain alignment by directly addressing the task of unlabeled target domain classification using CTDR achieves state-of-the-art results on eight visual and four language benchmark domain adaptation datasets.
\end{abstract}

Keywords-computer vision; deep learning; domain adaptation; sentiment analysis; transfer learning; unsupervised learning;

\section{INTRODUCTION}

The recent success of deep neural networks in supervised learning tasks over several areas like computer vision, speech, natural language processing can be attributed to the models that are trained on large amounts of labeled data. However, acquiring large amounts of labeled data in some domains can be very expensive or not possible at all. Additionally, the amount of time required for labeling the data to use existing deep learning techniques can be very high initially for the new domain. This is referred as coldstart. On the contrary, cost-effective unlabeled data can be easily obtained in large amounts for most new domains. So, one can aim to transfer the knowledge from a labeled source domain to perform tasks on an unlabeled target domain.

To study this, under the purview of transductive transfer learning, several approaches like domain adaptation, sample selection bias, co-variance shift have been explored in recent times. In this work, we study unsupervised domain adaptation by learning contrastive features in the unlabeled target domain in a fully unsupervised manner utilizing preexisting informative knowledge from the labeled source domain.Existing domain adaptation approaches mostly rely on domain alignment, i.e., align both domains so that they are superimposed and indistinguishable. This domain alignment can be achieved in three main ways: (a) discrepancy-based methods [1]-[5], (b) reconstruction-based methods [6], [7], and (c) adversarial adaptation methods [8]-[19].

Unlike above methods, our main motivation comes from the human ability to 'contradistinguish' and the fundamental idea of statistical learning as described by V. Vapnik [20] that indicates any desired problem should be tried to solve in a most possible direct way rather than solving a more general intermediate task. In the context of domain adaptation, the desired problem is classification on the unlabeled target domain and domain alignment followed by most standard methods is the general intermediate. This motivates us to propose an approach that does not require domain alignment. Our main contributions in this paper are as follows:

1) We propose a simple method that directly addresses the problem of domain adaptation by learning a single classifier, which we refer to as Contradistinguisher (CTDR), jointly in an unsupervised manner over the unlabeled target space and in a supervised manner over the labeled source space. Hence, overcoming the drawbacks of distribution alignment based techniques.

2) We formulate a 'contradistinguish loss' to directly utilize unlabeled target domain and address the classification task using unsupervised feature learning. A similar approach called DisCoder [21] was used for a much simpler task of semi-supervised feature learning on a single domain with no domain distribution shift.

3) From our experiments, we show that by jointly training CTDR on the source and target domain distributions, we can achieve above/on-par results over several methods. Surprisingly, this simple method re- 
sults in improvement over the state-of-the-art for eight challenging benchmark datasets in visual domains (USPS [22], MNIST [23], SVHN [24], SYNNUMBERS [8], CIFAR-10 [25], STL-10 [26], SYNSIGNS [8] and GTSRB [27]) and four benchmark language domains (Books, DVDs, Electronics, and Kitchen Appliances) of Amazon customer reviews sentiment analysis dataset [28].

The rest of the paper is structured as follows. Section II discusses on related works in domain adaptation. In Section III. we discuss the problem formulation, architecture, loss function definitions, algorithms, and complexity analysis of our proposed method CUDA. Section IV deals with the discussion of the experimental setup, results and analysis on vision and language domains. Finally in Section V, we conclude by highlighting the key contributions of CUDA.

\section{RELATED WORK}

As mentioned earlier, almost all domain adaptation approaches rely on domain alignment techniques. Here we briefly discuss three main techniques of domain alignment.

(a) Discrepancy-based methods: Associative Domain Adaptation (ADA) [1] learns statistically domain invariant embeddings using association loss as an alternative to Maximum Mean Discrepancy (MMD) [29]. Maximum Classifier Discrepancy (MCD) [2] aligns source and target distributions by maximizing the discrepancy between two separate classifiers. Self Ensembling (SE) [3] uses mean teacher variant [30] of temporal ensembling [31] with heavy reliance on data augmentation to minimize the discrepancy between student and teacher network predictions. Variational Fair Autoencoder (VFAE) [4] uses Variational Autoencoder (VAE) [32] with MMD to obtain domain invariant features. Central Moment Discrepancy (CMD) [5] proposes to match higher order moments of source and target domain distributions.

(b) Reconstruction-based methods: Deep ReconstructionClassification Networks (DRCN) [6] and Domain Separation Networks (DSN) [7] approaches learn a shared encodings of source and target domains using reconstruction networks.

(c) Adversarial adaptation methods: Reverse Gradient (RevGrad/DANN) [8], [33] uses domain discriminator to learn domain invariant representations of both the domains. Coupled Generative Adversarial Network (CoGAN) [9] uses Generative Adversarial Network (GAN) [34] to obtain domain invariant features used for classification. Adversarial Discriminative Domain Adaptation (ADDA) [10] uses GANs along with weight sharing to learn domain invariant features. Generate to Adapt (G2A) [11] learns to generate equivalent image in the other domain for a given image, thereby learning common domain invariant embeddings. Cross-Domain Representation Disentangler (CDRD) [12] learns cross-domain disentangled features for domain adaptation. Symmetric Bi-Directional Adaptive GAN (SBADA-
GAN) [13] aims to learn symmetric bidirectional mappings among the domains by trying to mimic a target image given a source image. Cycle-Consistent Adversarial Domain Adaptation (CyCADA) [14] adapts representations at both the pixel-level and feature-level over the domains. Moving Semantic Transfer Network (MSTN) [15] proposes moving semantic transfer network that learn semantic representations for the unlabeled target samples by aligning labeled source centroids and pseudo-labeled target centroids. Conditional Domain Adversarial Network (CDAN) [16] conditions the adversarial adaptation models on discriminative information conveyed in the classifier predictions. Joint Discriminative Domain Adaptation (JDDA) [17] proposes joint domain alignment along with discriminative feature learning. Decision-boundary Iterative Refinement Training with a Teacher (DIRT-T) [18] and Augmented Cyclic Adversarial Learning (ACAL) [19] learn by using a domain discriminator along with data augmentation for domain adaptation.

Apart from these standard ways, a slight deviant method explored is Tri-Training. Tri-Training algorithms use three classifiers trained on the labeled source domain and refine them for unlabeled target domain. To be precise, in each round of tri-training, a target sample is pseudo-labeled if the other two classifiers agree on the labeling, under certain conditions such as confidence thresholding. Asymmetric TriTraining (ATT) [35] uses three classifiers to bootstrap high confidence target domain samples by confidence thresholding. This way of bootstrapping works only if the source classifier has very high accuracy. In case of of low source classifier accuracy, target samples are never obtained to bootstrap, resulting in a bad model. Multi-Task Tri-training (MT-Tri) [36] explores the tri-training technique on the language domain adaptation tasks.

All the domain adaptation approaches mentioned earlier have a common unifying theme: they attempt to morph the target and source distributions so as to make them indistinguishable. Once the two distributions are perfectly aligned, they use a classifier trained on labeled source domain to classify the unlabeled target domain. Hence, the performance of the classifier on the target domain depends crucially on the domain alignment. As a result, the actual task of target domain classification is solved indirectly using domain alignment rather than using the unlabeled target data in an unsupervised manner which is a more logical and direct way.

In this paper, we propose a completely different approach: instead of focusing on aligning the source and target distributions, we learn a single classifier referred as Contradistinguisher (CTDR), jointly on both the domain distributions using contradistinguish loss for the unlabeled target data and supervised loss for the labeled source data. 


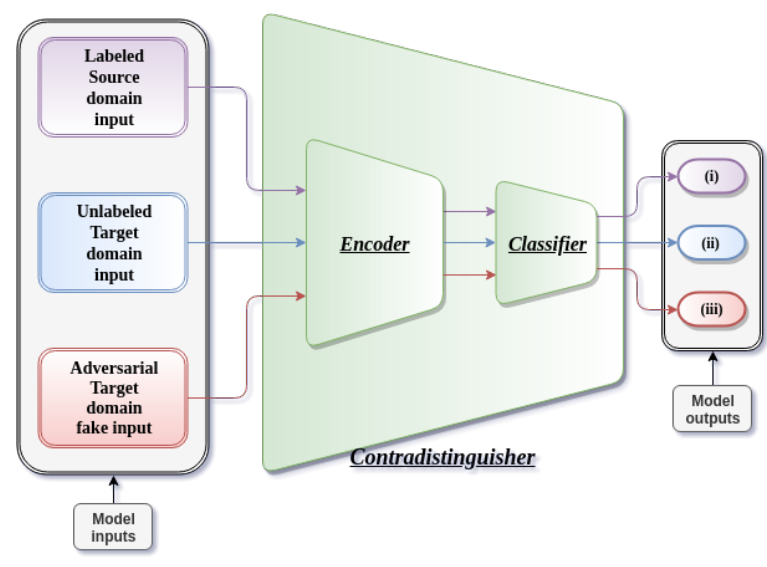

Figure 1. Architecture of the proposed method CUDA with Contradistinguisher (Encoder and Classifier). Three optimization objectives with their respective inputs involved in training of CUDA: (i) Source supervised 22, (ii) Target unsupervised (5), and Adversarial regularization (9).

\section{Proposed Method: CUDA}

A domain $\mathcal{D}_{d}$ is specified by its input feature space $\mathcal{X}_{d}$, the label space $\mathcal{Y}_{d}$ and the joint probability distribution $p\left(\mathbf{x}_{d}, \mathbf{y}_{d}\right)$, where $\mathbf{x}_{d} \in \mathcal{X}_{d}$ and $\mathbf{y}_{d} \in \mathcal{Y}_{d}$. Let $\left|\mathcal{Y}_{d}\right|=K$ be the number of class labels such that $\mathbf{y}_{d} \in\{0, \ldots, K-1\}$ for any instance $\mathbf{x}_{d}$. In particular, Domain adaptation consists of two domains $\mathcal{D}_{s}$ and $\mathcal{D}_{t}$ that are referred as the source and target domains respectively. A common assumption in domain adaptation is that the input feature space as well as the label space remains unchanged across the source and the target domain, i.e., $\mathcal{X}_{s}=\mathcal{X}_{t}=\mathcal{X}_{d}$ and $\mathcal{Y}_{s}=\mathcal{Y}_{t}=\mathcal{Y}_{d}$. Hence, the only difference between the source and target domain is input-label space distributions, i.e., $p\left(\mathbf{x}_{s}, \mathbf{y}_{s}\right) \neq p\left(\mathbf{x}_{t}, \mathbf{y}_{t}\right)$. This is referred as domain shift in the standard literature of domain adaptation.

In particular, in an unsupervised domain adaptation, the training data consists of labeled source domain instances $\left\{\left(\mathbf{x}_{s}^{i}, \mathbf{y}_{s}^{i}\right)\right\}_{i=1}^{n_{s}}$ and unlabeled target domain instances $\left\{\mathbf{x}_{t}^{j}\right\}_{j=1}^{n_{t}}$. Given a labeled data in the source domain, it is straightforward to learn a classifier by maximizing the conditional probability $p\left(\mathbf{y}_{s} \mid \mathbf{x}_{s}\right)$ over the labeled samples. However, the task at hand is to learn a classifier on the unlabeled target domain by transferring the knowledge from the labeled source domain.

\section{A. Overview}

Figure 1 indicates the model architecture of our proposed method CUDA, i.e., Contradistinguisher (CTDR) and the respective losses involved in CUDA training.

The objective of CTDR is to find a clustering scheme using the most contrastive features on unlabeled target in such a way that it also satisfies the target domain prior, i.e., prior enforcing. We achieve this by jointly training labeled source samples in a supervised manner and unlabeled target samples in an unsupervised end-to-end manner by using a contradistinguish loss same as [21]. This finetunes the classifier learnt from source domain to the target domain. The main important feature of our approach is the contradistinguish loss (5) which is discussed in detail in Section $\amalg I I-C$.

Note that the objective of the CTDR is not same as a classifier, i.e., distinguishing is not same as classifying. Suppose there are two contrastive entities $e_{1} \in C_{1}$ and $e_{2} \in C_{2}$, where $C_{1}, C_{2}$ are two classes. The aim of a classifier is to classify $e_{1} \in C_{1}$ and $e_{2} \in C_{2}$, where to train a classifier one requires labeled data. On the contrary, the job of a CTDR is to just identify $e_{1} \neq e_{2}$, i.e., CTDR can classify $e_{1} \in C_{1}$ (or $C_{2}$ ) and $e_{2} \in C_{2}$ (or $C_{1}$ ) indifferently. To train CTDR, we do not need any class information but only need unlabeled entities $e_{1}$ and $e_{2}$. Using unlabeled target data, CTDR is able to distinguish the samples in an unsupervised way. However, since the final task is classification, one would require a selective incorporation of the pre-existing informative knowledge required for the task of classification. This knowledge is obtained by jointly training, thus classifying $e_{1} \in C_{1}$ and $e_{2} \in C_{2}$.

In the subsequent Sections III-B [II-E we formulate the optimization objectives and also perform complexity analysis.

\section{B. Supervised Source Classification}

For the labeled source domain instances $\left\{\left(\mathbf{x}_{s}^{i}, \mathbf{y}_{s}^{i}\right)\right\}_{i=1}^{n_{s}}$, we define the conditional-likelihood of observing $\mathbf{y}_{s}$ given $\mathbf{x}_{s}$ as, $p_{\theta}\left(\mathbf{y}_{s} \mid \mathbf{x}_{s}\right)$, where $\theta$ denotes the parameters of CTDR.

We estimate $\theta$ by maximizing the conditional loglikelihood of observing the labels given the labeled source domain samples. The source domain supervised objective to maximize

$$
\mathcal{L}_{s}(\theta)=\sum_{i=1}^{n_{s}} \log \left(p_{\theta}\left(\mathbf{y}_{s}^{i} \mid \mathbf{x}_{s}^{i}\right)\right) .
$$

Alternatively, one can minimize the cross-entropy loss

$$
\mathcal{L}_{c e}(\theta)=-\sum_{i=1}^{n_{s}} \sum_{k=0}^{K-1} \mathbb{1}\left[\mathbf{y}_{s}^{i}=k\right] \log \left(\hat{\mathbf{y}}_{s}^{i k}\right),
$$

where $\hat{\mathbf{y}}_{s}^{i k}$ is the softmax output of CTDR that represents the probability of class $k$ for the given sample $\mathbf{x}_{s}^{i}$.

\section{Unsupervised Target Classification}

For the unlabeled target domain instances $\left\{\mathbf{x}_{t}^{j}\right\}_{j=1}^{n_{t}}$, as the corresponding labels are unknown, a naive way of predicting the target labels is to directly use the classifier trained only with supervised loss (2). Though this gives some good results, it fails to achieve high accuracies due to two reasons: (i) $p_{\theta}$ is defined over $\mathbf{x}_{s}$ and not $\mathbf{x}_{t}$. (ii) $p_{\theta}$ is not a valid probability distribution because $\sum_{\ell=1}^{n_{t}} p_{\theta}\left(\mathbf{y}_{t} \mid \mathbf{x}_{t}^{\ell}\right) \neq 1$. Enforcing these two conditions, we model a non-trivial joint 
distribution $\hat{q}_{\theta}\left(\mathbf{x}_{t}, \mathbf{y}_{t}\right)$ parameterized by $\theta$ over target domain as,

$$
\hat{q}_{\theta}\left(\mathbf{x}_{t}, \mathbf{y}_{t}\right)=\frac{p_{\theta}\left(\mathbf{y}_{t} \mid \mathbf{x}_{t}\right)}{\sum_{\ell=1}^{n_{t}} p_{\theta}\left(\mathbf{y}_{t} \mid \mathbf{x}_{t}^{\ell}\right)} .
$$

However (3) is not exactly a joint distribution yet because $\sum_{\ell=1}^{n_{t}} \hat{q}_{\theta}\left(\mathbf{x}_{t}^{\ell}, \mathbf{y}_{t}\right) \neq p\left(\mathbf{y}_{t}\right)$, i.e., marginalizing over all $\left\{\mathbf{x}_{t}^{j}\right\}_{j=1}^{n_{t}}$ should yield the target prior distribution $p\left(\mathbf{y}_{t}\right)$. We modify (3) so as to include the marginalization condition. We refer to this as target domain prior enforcing.

$$
q_{\theta}\left(\mathbf{x}_{t}, \mathbf{y}_{t}\right)=\frac{p_{\theta}\left(\mathbf{y}_{t} \mid \mathbf{x}_{t}\right) * p\left(\mathbf{y}_{t}\right)}{\sum_{\ell=1}^{n_{t}} p_{\theta}\left(\mathbf{y}_{t} \mid \mathbf{x}_{t}^{\ell}\right)} .
$$

Note that $q_{\theta}\left(\mathbf{x}_{t}, \mathbf{y}_{t}\right)$ defines a non-trivial approximate of joint distribution over the target domain as a function of $p_{\theta}$ learnt over source domain. The resultant unsupervised maximization objective for the target domain is given by maximizing the log-probability of the joint distribution $q_{\theta}\left(\mathbf{x}_{t}, \mathbf{y}_{t}\right)$ which is

$$
\mathcal{L}_{t}\left(\theta,\left\{\mathbf{y}_{t}^{j}\right\}_{j=1}^{n_{t}}\right)=\sum_{j=1}^{n_{t}} \log \left(q_{\theta}\left(\mathbf{x}_{t}^{j}, \mathbf{y}_{t}^{j}\right)\right) .
$$

Next, we discuss how the objective (5) is solved and the reason why (5) is referred as contradistinguish loss. Since the target labels $\left\{\mathbf{y}_{t}^{j}\right\}_{j=1}^{n_{t}}$ are unknown, one needs to maximize (5) over the parameters $\theta$ as well as the unknown target labels $\mathbf{y}_{t}$. As there are two parameters for maximization, we follow a two step approach to maximize (5). The two optimization steps are as follows.

(i) Pseudo-label selection: We maximize (5) only with respect to the label $\mathbf{y}_{t}$ for every $\mathbf{x}_{t}$ by fixing $\theta$ as

$$
\hat{\mathbf{y}}_{t}^{j}=\underset{\mathbf{y}^{j} \in \mathcal{Y}_{t}}{\arg \max } \frac{p_{\theta}\left(\mathbf{y}^{j} \mid \mathbf{x}_{t}^{j}\right) * p\left(\mathbf{y}_{t}\right)}{\sum_{\ell=1}^{n_{t}} p_{\theta}\left(\mathbf{y}^{\ell} \mid \mathbf{x}_{t}^{\ell}\right)} .
$$

Pseudo-labeling approach under semi-supervised representation learning setting has been well studied in [37] and shown equivalent to entropy regularization [38]. We derive the motivation from [21] that also use pseudo-labeling in the context of semi-supervised representation learning. However, our method addresses a more complex problem of domain adaptation in the presence of domain shift.

(ii) Maximization: By fixing the pseudo-labels $\left\{\hat{\mathbf{y}}_{t}^{j}\right\}_{j=1}^{n_{t}}$ from (6), we train CTDR to maximize (5) with respect to the parameter $\theta$.

$$
\begin{aligned}
\mathcal{L}_{t}(\theta)= & \sum_{j=1}^{n_{t}} \log \left(p_{\theta}\left(\hat{\mathbf{y}}_{t}^{j} \mid \mathbf{x}_{t}^{j}\right)\right)+\sum_{j=1}^{n_{t}} \log \left(p\left(\mathbf{y}_{t}\right)\right) \\
& -\sum_{j=1}^{n_{t}} \log \left(\sum_{\ell=1}^{n_{t}} p_{\theta}\left(\hat{\mathbf{y}}_{t}^{\ell} \mid \mathbf{x}_{t}^{\ell}\right)\right) .
\end{aligned}
$$

The first term, i.e., log-probability for a given $\mathbf{x}_{t}^{j}$ forces CTDR to choose features to classify $\mathbf{x}_{t}^{j}$ to $\hat{\mathbf{y}}_{t}^{j}$. The second term is a constant, hence it has no effect in optimization with respect to $\theta$. The third term is the negative of log-probability for all the samples $\mathbf{x}_{t}$ in the entire domain. Maximization of this term forces CTDR to choose features to not classify all the other $\mathbf{x}_{t}^{\ell \neq j}$ to selected pseudo-label $\hat{\mathbf{y}}_{t}^{j}$ except the given sample $\mathbf{x}_{t}^{j}$. This forces CTDR to extract the most unique features of a given sample $\mathbf{x}_{t}^{j}$ against all the other samples $\mathbf{x}_{t}^{\ell \neq j}$, i.e., most unique contrastive feature of the selected sample $\mathbf{x}_{t}^{j}$ over all the other samples $\mathbf{x}_{t}^{\ell \neq j}$ to distinguish a given sample from all others.

The first and third term together in (7) enforce that CTDR learns the most contradistinguishing features among the samples $\mathbf{x}_{t} \in \mathcal{X}_{t}$, thus performing unlabeled target domain classification in a fully unsupervised way. Because of this contradistinguishing feature learning, we refer the unsupervised target domain objective (5) as contradistinguish loss.

Ideally, one would like to compute the third term in (7) using the complete target training data for each input sample. Since it is expensive to compute the third term over the entire $\mathbf{x}_{t}$ for each individual sample during training, one evaluates the third term in (7) over a mini-batch. In our experiments, we have observed that mini-batch strategy does not cause any problem during training as far as it includes at least one sample from each class which is guaranteed for a reasonably large mini-batch size of 128 . For numerical stability, we use $\log \sum \exp$ trick to optimize third term in (7).

\section{Adversarial Regularization}

In order to prevent CTDR from over-fitting to the chosen pseudo labels during the training, we use adversarial regularization. In particular, we train CTDR to be confused about set of fake - ve samples $\left\{\hat{\mathbf{x}}_{t}^{j}\right\}_{j=1}^{n_{f}}$ by maximizing the conditional log-probability over the given fake sample such that the sample belongs to all $K\left(\left|\mathcal{Y}_{d}\right|\right)$ classes simultaneously. The objective of the adversarial regularization is to multilabel the fake sample (e.g., noisy image that looks like a cat and a dog) equally to all $K$ classes as labeling to any unique class introduces more noise in pseudo labels. This strategy is similar to entropy regularization [38] in the sense that instead of minimizing the entropy for the real target samples, we maximize the conditional log-probability over the fake -ve samples. Therefore, we add the following maximization objective to the total CTDR objective as a regularizer.

$$
\mathcal{L}_{a d v}(\theta)=\sum_{j=1}^{n_{f}} \log \left(p_{\theta}\left(\hat{\mathbf{y}}_{t}^{j} \mid \hat{\mathbf{x}}_{t}^{j}\right)\right),
$$

for all $\hat{\mathbf{y}}_{t}^{j} \in \mathcal{Y}_{t}$. As maximization of $(8)$ is analogous to minimize the binary cross-entropy loss 9 of a multi-class multilabel classification task, in our practical implementation, we minimize (9) for assigning labels to all the classes for every samples.

$$
\mathcal{L}_{b c e}(\theta)=-\sum_{j=1}^{n_{f}} \sum_{k=0}^{K-1} \log \left(\hat{\mathbf{y}}_{t}^{j k}\right)
$$


where $\hat{\mathbf{y}}_{t}^{j k}$ is the softmax output of CTDR which represents the probability of class $k$ for the given sample $\hat{\mathbf{x}}_{t}^{j}$.

The fake $-v e$ samples $\hat{\mathbf{x}}_{t}$ can be directly sampled from, say a Gaussian distribution in the input feature space $\mathcal{X}_{t}$ with the mean and standard deviation of the samples $\mathrm{x}_{t} \in \mathcal{X}_{t}$. For the language domain, fake samples are generated randomly as mentioned above. In case of image datasets, as the feature space is high dimensional, the fake images $\hat{\mathbf{x}}_{t}$ are generated using a generator network $G_{\phi}$ with parameter $\phi$ that takes Gaussian noise vector $\eta_{t}$ as input to produce a fake sample $\hat{\mathbf{x}}_{t}$, i.e., $\hat{\mathbf{x}}_{t}=G_{\phi}\left(\eta_{t}\right)$. Generator $G_{\phi}$ is trained by minimizing kernel MMD loss [39], i.e., a modified version of MMD loss between the encoder output $\rho\left(\hat{\mathbf{x}}_{t}\right)$ and $\rho\left(\mathbf{x}_{t}\right)$ of $n_{f}$ fake images $\hat{\mathbf{x}}_{t}$ and $n_{t}$ real target domain images $\mathbf{x}_{t}$ respectively.

$$
\begin{aligned}
\mathcal{L}_{g e n}(\phi)= & \frac{1}{n_{f}^{2}} \sum_{i=1}^{n_{f}} \sum_{j=1}^{n_{f}} k\left(\rho\left(\hat{\mathbf{x}}_{t}^{i}\right), \rho\left(\hat{\mathbf{x}}_{t}^{j}\right)\right) \\
& +\frac{1}{n_{t}^{2}} \sum_{i=1}^{n_{t}} \sum_{j=1}^{n_{t}} k\left(\rho\left(\mathbf{x}_{t}^{i}\right), \rho\left(\mathbf{x}_{t}^{j}\right)\right) \\
& -\frac{2}{n_{t} * n_{f}} \sum_{i=1}^{n_{f}} \sum_{j=1}^{n_{t}} k\left(\rho\left(\hat{\mathbf{x}}_{t}^{i}\right), \rho\left(\mathbf{x}_{t}^{j}\right)\right),
\end{aligned}
$$

where $k\left(x, x^{\prime}\right)=e^{-\gamma\left\|x-x^{\prime}\right\|^{2}}$ is the Gaussian kernel.

Note that the objective of the generator is not to generate realistic image but to generate fake noisy images with mixed image attributes from the target domain. This reduces the effort of training powerful generators which is the focus in adversarial based domain adaptation approaches [11] $-[15]$ used for domain alignment.

\section{E. Algorithms and Complexity Analysis}

Algorithm 1 and 2 list steps involved in CUDA training and inference respectively. Here we briefly discuss time complexity of Algorithm 1 and 2 We also compare model complexity of CUDA against domain alignment approaches.

(a) Time complexity: We consider a batch of $b$ instances for forward and backward propagation during training. For source supervised loss (2), the time complexity is $O\left(b K T_{c}\right)$, where $T_{c}$ is the time complexity involved in obtaining the classifier output which mainly depends on the model complexity which will be discussed next. For target unsupervised loss [5], the time complexity is $O\left(b K T_{c}\right)$ for pseudo-label selection and $O\left(b K T_{c}+b^{2} K T_{c}\right)$ for first and third terms in maximization step, i.e., $O\left(b^{2} K T_{c}\right)$ effectively for the target unsupervised loss (5). The adversarial regularization loss (9) complexity corresponds to $O\left(b K T_{c}\right)$. Time complexity for generator training is $O\left(b^{2} D_{e} T_{e}\right)$, where $D_{e}$ is dimension of the encoder output and $T_{e}$ is the time complexity for the encoder output from neural network which also depends on the model complexity discussed next. As $T_{c}$ dominates $T_{e}$, total training time complexity can be further simplified to
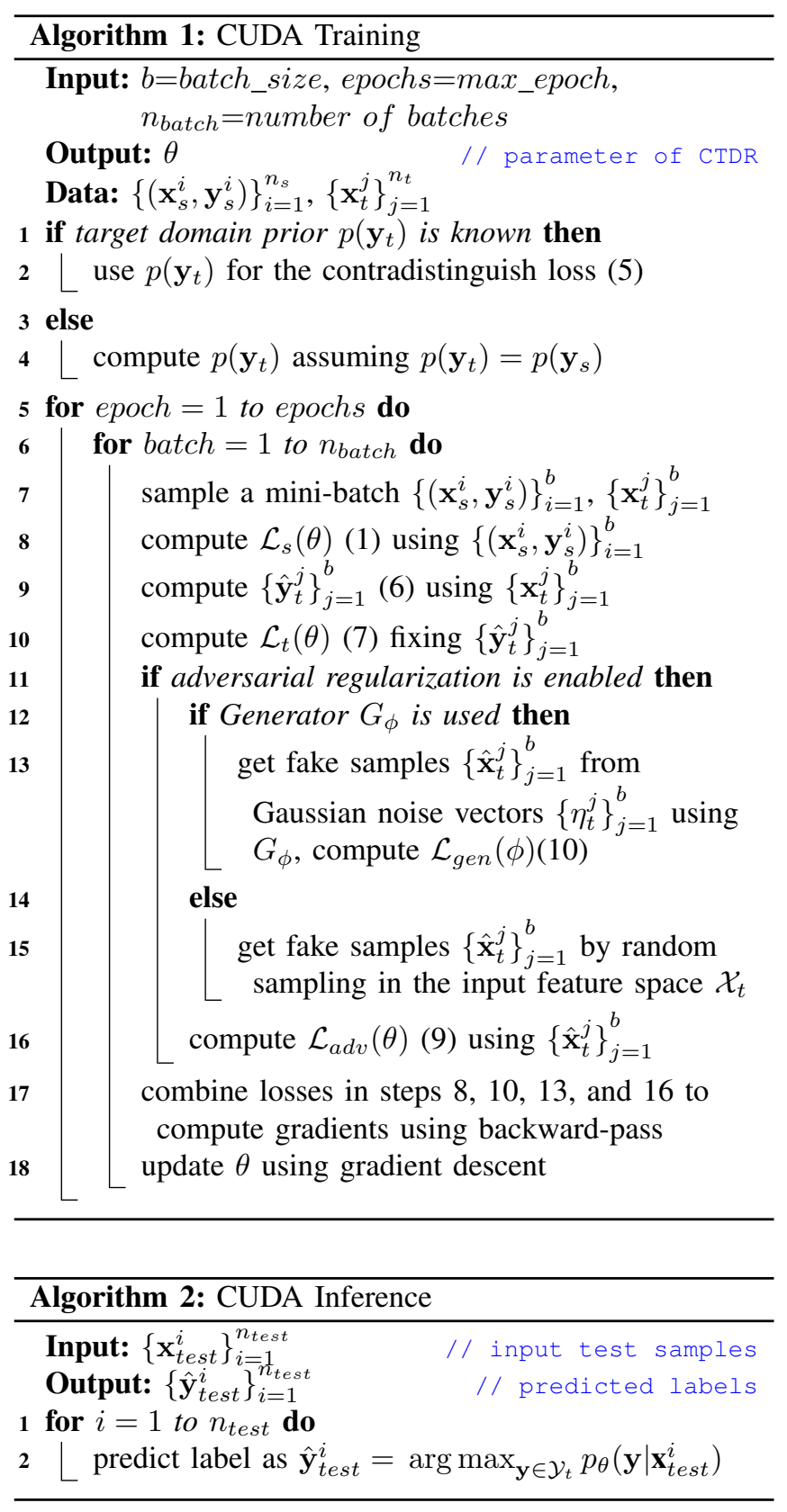

$O\left(b^{2} K T_{c}\right)$. During inference phase, the time complexity is $O\left(n_{\text {test }} T_{c}\right)$, where $n_{\text {test }}$ is the number of inference samples.

(b) Model complexity: As discussed above, $T_{c}$ mainly depends on the model complexity involving many factors such as input feature dimension, number of neural network layers, type of normalization, type of activation functions etc. CTDR is a simple network with a single encoder and classifier unlike MCD-DA that uses a single encoder with two classifier. This makes MCD-DA time complexity $2 T_{c}$ instead of just $T_{c}$. Similarly, SE uses 2 copies of network of encoder and classifier one for student and other for teacher network. This makes SE time complexity $2 T_{c}$ instead 
Table I

Table II

DETAILS OF LANGUAGE DATASET (AMAZON CUSTOMER REVIEWS FOR SENTIMENT ANALYSIS).

\begin{tabular}{lrrrlcc}
\hline Dataset & \# Train & \# Test & \# Classes & Target & Resolution & Channels \\
\hline USPS $(\mathcal{U S})$ & 7,291 & 2,007 & 10 & Digits & $16 \times 16$ & Mono \\
MNIST $(\mathcal{M N})$ & 60,000 & 10,000 & 10 & Digits & $28 \times 28$ & Mono \\
SVHN $(\mathcal{S V})$ & 73,257 & 26,032 & 10 & Digits & $32 \times 32$ & RGB \\
SYNNUMBERS $(\mathcal{S N})$ & 479,400 & 9,553 & 10 & Digits & $32 \times 32$ & RGB \\
\hline CIFAR-9 $(\mathcal{C} 9)$ & 45,000 & 9,000 & 9 & Object ID & $32 \times 32$ & RGB \\
STL-9 $(\mathcal{S} 9)$ & 4,500 & 7,200 & 9 & Object ID & $96 \times 96$ & RGB \\
\hline SYNSIGNS $(\mathcal{S S})$ & 100,000 & - & 43 & Traffic Signs & $40 \times 40$ & RGB \\
GTSRB $(\mathcal{G})$ & 39,209 & 12,630 & 43 & Traffic Signs & varies & RGB \\
\hline
\end{tabular}

1) Visual Domain Adaptation: We consider eight benchmark visual datasets with 3 different nature of images for our visual domain experiments. (a) Digits: USPS $(\mathcal{U S})[22]$ and MNIST $(\mathcal{M N})[23]$ are a pair of gray-scale digits datasets. SVHN $(\mathcal{S V})[24]$ and SYNNUMBERS $(\mathcal{S N})$ [8] are another pair of RGB digits datasets. (b) Objects: CIFAR $(\mathcal{C} 9)$ [25] and STL $(\mathcal{S} 9)[26]$ are a dataset pair of objects/animals RGB images by considering only the 9 overlapping classes from the original datasets. (c) Traffic Signs: SYNSIGNS $(\mathcal{S S})[8$ and GTSRB $(\mathcal{G T})$ [27] are a dataset pair with traffic signs. Table I provides visual dataset details and Figure 2 indicates some random samples from all eight datasets.

On these datasets, we consider eight main domain adaptation tasks studied in [3], [8]. These eight visual tasks and the data processing considered are as follows, (i) $\mathcal{U S} \leftrightarrow \mathcal{M N}: \mathcal{U S}$ images are up-scaled using bi-linear interpolation from $16 \times 16 \times 1$ to $28 \times 28 \times 1$ to match the size of $\mathcal{M N}$, (ii) $\mathcal{S} \mathcal{V} \leftrightarrow \mathcal{M N}: \mathcal{M N}$ images are up-scaled using bi-linear interpolation to $32 \times 32 \times 1$. The RGB channels of $\mathcal{S} \mathcal{V}$ are converted to Mono image resulting in $32 \times 32 \times 1$ size. Several other combinations were tried and this was chosen since the results are the best, (iii) $\mathcal{S N} \rightarrow \mathcal{S} \mathcal{V}:$ No preprocessing required as these domains have same image size, (iv) $\mathcal{C} 9 \leftrightarrow \mathcal{S} 9$ : Only the 9 overlapping classes from datasets as the label space should be same for both the domain. $\mathcal{S} 9$ images are down-scaled from $96 \times 96 \times 3$ to $32 \times 32 \times 3$ to match the size of $\mathcal{C} 9$. (v) $\mathcal{S S} \rightarrow \mathcal{G T}$ : Crop the images to $40 \times 40 \times 3$ based on the region of interest in the images in both datasets.

Note that we do not perform any image data augmentation in our experiments unlike [3]. Our aim in this paper is to demonstrate that the proposed method performs above/onpar without data augmentation as data augmentation is expensive and not always possible as seen in language tasks.

2) Language Domain Adaptation: We consider four benchmark language domains (i) Books $(\mathcal{B})$, (ii) DVDs $(\mathcal{D})$, (iii) Electronics $(\mathcal{E})$, and (iv) Kitchen Appliances $(\mathcal{K})$ from Amazon customer reviews [28] dataset. The dataset includes product reviews in four different domains for sentiment analysis as indicated in Table II

\section{A. Experimental Setup}




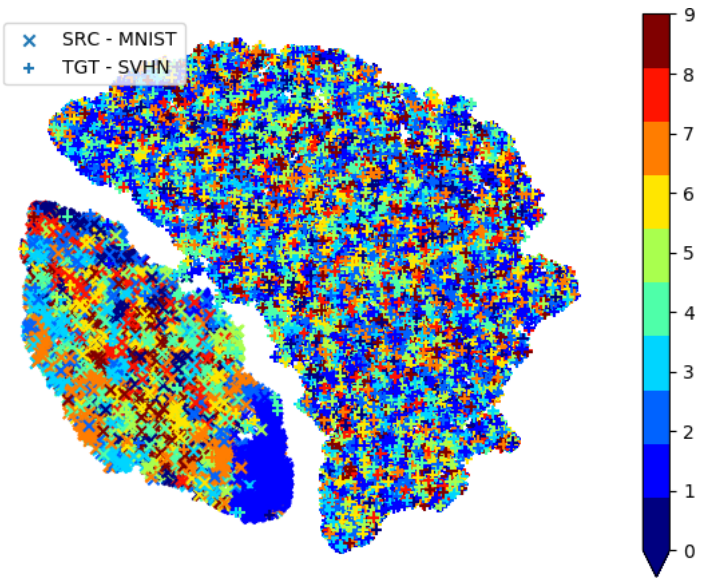

(a) Before training

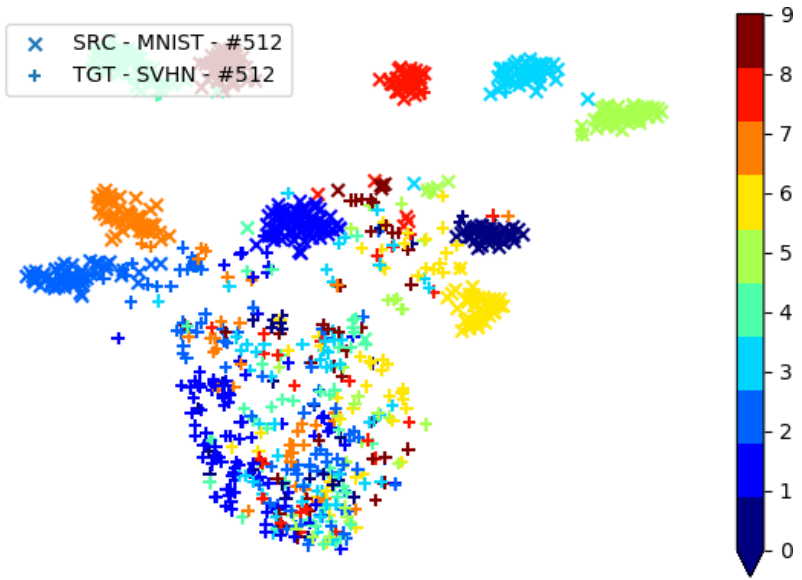

(c) after 6 epochs training

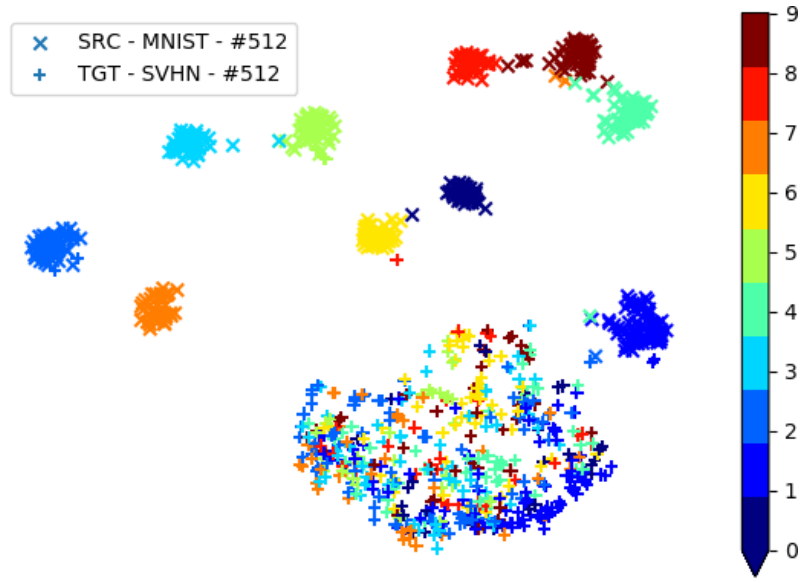

(b) after 1 epoch training

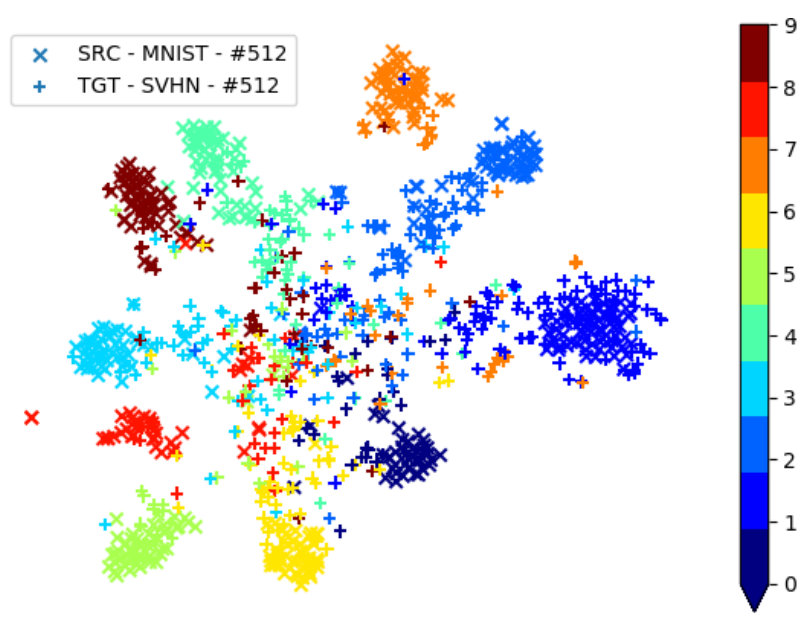

(d) after full training

Figure 3. t-SNE [40] plots for embeddings from the output of CTDR before applying softmax corresponding to the test samples from $\mathcal{M N} \rightarrow \mathcal{S V}$ visual task trained with CUDA. We consider this task as this is the most difficult among all the visual experiments due contrasting domains with high domain shift. (a) Initial plot of all the test samples before training indicating domain shift as there are two separate clusters for each domain. (b) Plot of subset from test samples after epoch=1. (c) Plot of subset from test samples after epoch=6. (d) Plot of subset from test samples after full CUDA training.

On these domains, we consider all twelve tasks studied in [4], [5], [33], [35], [36]. We use the same neural networks and text pre-processing used in [33], [36], [41] to get 5000 dimensional feature vector. We assign binary label ' 0 ' for the products rated from $\leq 3$ stars and ' 1 ' for $\geq 4$ star ratings.

We select the best existing neural networks without major modifications to hyper-parameters so as to demonstrate the effectiveness of CUDA. All the experiments are done using PyTorch [42] with mini-batch size of 64 per GPU distributed over four GPUs, Adam optimizer with an initial learning rate 0.001 and decay rate of 0.6 every 30 epochs.

\section{B. Experimental Results}

We use the same metric used for evaluation as in [1][19], [33], [35], [36], i.e., the accuracy on target domain test set. Table III indicates the target domain test accuracy across all the eight main domain adaptation tasks compared with several state-of-the-art domain alignment methods [1][3], [6]-[19], [35]. Table IV] indicates the target domain test accuracy across all the twelve domain adaptation tasks compared with different state-of-the-art methods [4], [5], [33], [35], [36].

Apart from the standard domain alignment methods used for comparison, we report two baselines $\mathcal{B L}_{1}$ and $\mathcal{B L} \mathcal{L}_{2}$ of our own, reported in Tables III and IV by fixing the CTDR neural network architecture and varying only the training losses used to demonstrate the effectiveness of CUDA. $\mathcal{B} \mathcal{L}_{1}$ indicates training CTDR using only the target domain in a fully supervised way. $\mathcal{B} \mathcal{L}_{2}$ indicates training CTDR using only the source domain in a fully supervised way. $\mathcal{B L}_{1}$ and $\mathcal{B} \mathcal{L}_{2}$ respectively indicates the maximum and minimum target domain test accuracy that can be attained with chosen 


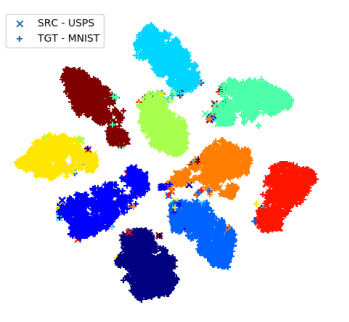

(a) USPS $\rightarrow$ MNIST

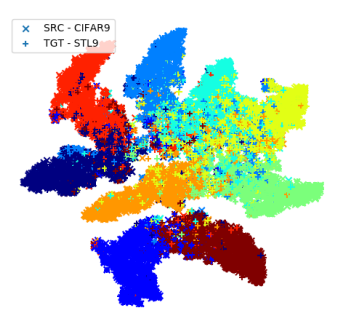

(e) CIFAR-9 $\rightarrow$ STL-9

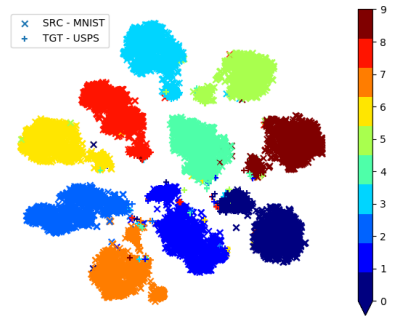

(b) MNIST $\rightarrow$ USPS

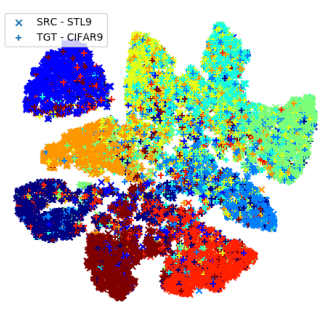

(f) STL-9 $\rightarrow$ CIFAR-9

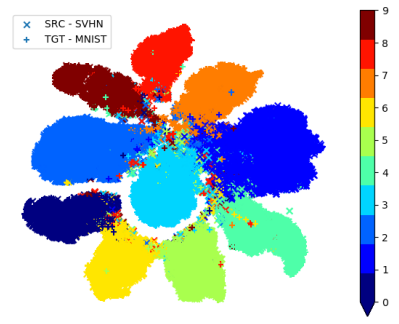

(c) $\mathrm{SVHN} \rightarrow \mathrm{MNIST}$
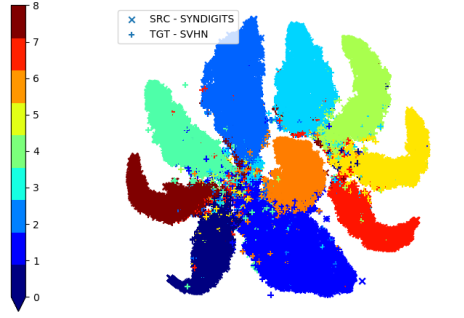

(g) SYNNUMBERS $\rightarrow$ SVHN

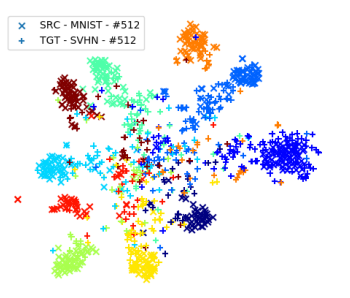

(d) $\mathrm{MNIST} \rightarrow \mathrm{SVHN}$

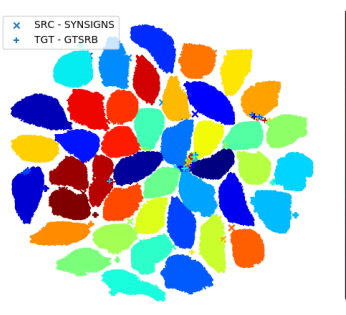

(h) SYNSIGNS $\rightarrow$ GTSRB

Figure 4. t-SNE [40] plots for embeddings from the output of CTDR before applying softmax corresponding to the test samples in visual experiments.

Table III

TARGET DOMAIN TEST ACCURACY (\%) ON IMAGE DATASETS. CUDA CORRESPONDS TO OUR BEST RESULTS OBTAINED WITH BEST HYPER-PARAMETER SETTINGS. $t s=\mathcal{B} \mathcal{L}_{1}$ : TARGET SUPERVISED, $s s=\mathcal{B L}_{2}$ : SOURCE SUPERVISED, $t u$ : TARGET UNSUPERVISED, $s u$ : SOURCE UNSUPERVISED, $t a$ : ADVERSARIAL REGULARIZATION AND $s a$ : SOURCE ADVERSARIAL REGULARIZATION REPRESENTS DIFFERENT TRAINING CONFIGURATIONS. WE EXCLUDE [3], [18], [19] FROM COMPARISON AS THEY USE HEAVY DATA AUGMENTATION.

\begin{tabular}{|c|c|c|c|c|c|c|c|c|}
\hline Method & $\mathcal{U S} \rightarrow \mathcal{M N}$ & $\mathcal{M N} \rightarrow \mathcal{U S}$ & $\mathcal{S V} \rightarrow \mathcal{M N}$ & $\mathcal{M N} \rightarrow \mathcal{S V}$ & $\mathcal{C} 9 \rightarrow \mathcal{S} 9$ & $\mathcal{S} 9 \rightarrow \mathcal{C} 9$ & $\mathcal{S N} \rightarrow \mathcal{S V}$ & $\mathcal{S S} \rightarrow \mathcal{G T}$ \\
\hline ADA $\sqrt{1}$ & - & - & 97.16 & - & - & - & 91.86 & 97.66 \\
\hline MCD-DA 2 & 94.10 & 94.20 & 96.20 & - & - & - & - & 94.40 \\
\hline DRCN $|6|$ & 73.67 & 91.80 & 81.97 & 40.05 & 66.37 & 58.65 & - & - \\
\hline $\mathrm{DSN}$ & - & - & 82.70 & - & - & - & 91.20 & 93.10 \\
\hline RevGrad [8] & 74.01 & 91.11 & 73.91 & 35.67 & 66.12 & 56.91 & 91.09 & 88.65 \\
\hline CoGAN $[9]$ & 89.10 & 91.20 & - & - & - & - & - & - \\
\hline ADDA 10 & 90.10 & 89.40 & 76.00 & - & - & - & - & - \\
\hline $\mathrm{G} 2 \mathrm{~A} \mid 11$ & 90.80 & 92.50 & 84.70 & 36.40 & - & - & - & - \\
\hline CDRD [12 & 94.35 & 95.05 & - & - & - & - & - & - \\
\hline SBADA-GAN [13] & 95.00 & 97.60 & 76.10 & 61.10 & - & - & - & 96.70 \\
\hline CyCADA $\mid 14]$ & 96.50 & 95.60 & 90.40 & - & - & - & - & - \\
\hline MSTN & - & 92.90 & 91.70 & - & - & - & - & - \\
\hline CDAN & 97.10 & 96.50 & 90.50 & - & - & - & - & - \\
\hline JDDA & 96.70 & - & 94.20 & - & - & - & - & - \\
\hline ATT $|35|$ & - & - & 86.20 & 52.80 & - & - & 93.10 & 96.20 \\
\hline CUDA (Ours) & 99.20 & 97.86 & 99.07 & 71.30 & 77.22 & 65.93 & 94.30 & 99.40 \\
\hline$t s=\mathcal{B} \mathcal{L}_{1}$ (Ours) & 99.64 & 97.98 & 99.64 & 96.02 & 73.78 & 91.46 & 96.85 & 98.23 \\
\hline$s s=\mathcal{B L}_{2}$ (Ours) & 81.18 & 82.00 & 77.54 & 24.86 & 77.64 & 62.10 & 91.45 & 95.13 \\
\hline$s s+t u$ (Ours) & 98.83 & 97.71 & 98.81 & 50.83 & 77.22 & 62.50 & 93.65 & 98.15 \\
\hline$s s+t u+s u$ (Ours) & 98.77 & 97.86 & 98.62 & 54.38 & 76.93 & 61.09 & 93.52 & 97.86 \\
\hline$s s+t u+s u+t a$ (Ours) & 99.20 & 97.31 & 98.85 & 54.32 & 76.18 & 59.37 & 93.59 & 99.40 \\
\hline$s s+t u+s u+s a$ (Ours) & 89.97 & 93.87 & 97.15 & 41.71 & 75.00 & 56.99 & 90.79 & 99.35 \\
\hline$s s+t u+s u+s a+t a$ (Ours) & 98.75 & 96.26 & 95.73 & 55.25 & 70.93 & 61.37 & 92.97 & 99.11 \\
\hline SE [3] & 99.54 & 98.26 & 99.26 & 97.00 & 80.09 & 74.24 & 97.11 & 99.37 \\
\hline DIRT-T [18] & - & - & 99.40 & 54.50 & - & 73.30 & 96.20 & 99.60 \\
\hline ACAL 19 & 97.16 & 98.31 & 96.51 & 60.85 & - & - & 97.98 & - \\
\hline
\end{tabular}

CTDR neural network.

Comparing CUDA with $\mathcal{B L}_{2}$ in Tables III and IV we can see huge improvements in the target domain test accuracies due to the use of contradistinguish loss 5 demonstrating the effectiveness of CTDR.

As our method is mainly dependent on the contradistin- 


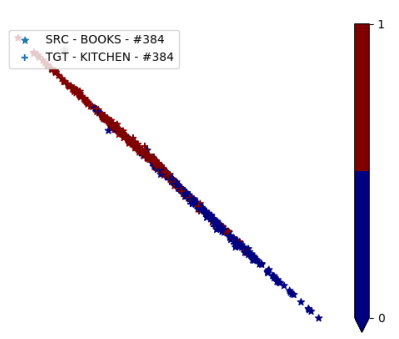

(a) $\mathcal{B} \rightarrow \mathcal{K}$

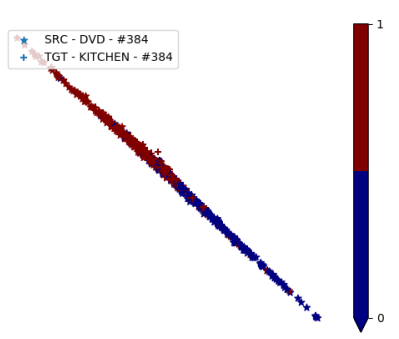

(b) $\mathcal{D} \rightarrow \mathcal{K}$

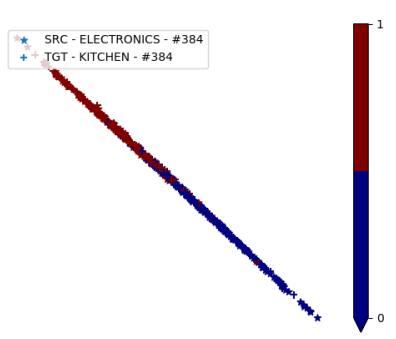

(c) $\mathcal{E} \rightarrow \mathcal{K}$

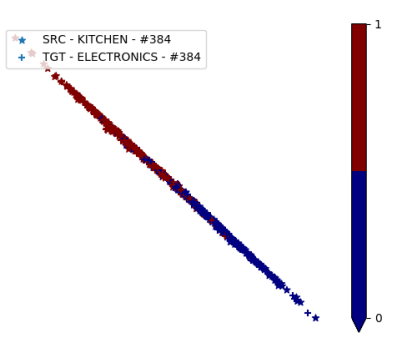

(d) $\mathcal{K} \rightarrow \mathcal{E}$

Figure 5. t-SNE [40] plots for embeddings from the output of CTDR corresponding to the test samples in language experiments . (Note: For the sake of the brevity, we do not add the plots for all the language tasks as language tasks plots are almost similar and not as informative as visual tasks.)

Table IV

TARGET DOMAIN TEST ACCURACY (\%) ON AMAZON CUSTOMER REVIEWS DATASET FOR SENTIMENT ANALYSIS. CUDA CORRESPONDS TO OUR BEST RESULTS OBTAINED WITH BEST HYPER-PARAMETER SETTINGS. $t s=\mathcal{B L}_{1}$ : TARGET SUPERVISED, $s s=\mathcal{B} \mathcal{L}_{2}$ : SOURCE SUPERVISED, $t u$ : TARGET UNSUPERVISED, $s u$ : SOURCE UNSUPERVISED AND $t a$ : ADVERSARIAL REGULARIZATION REPRESENTS DIFFERENT TRAINING CONFIGURATIONS.

\begin{tabular}{|c|c|c|c|c|c|c|c|c|c|c|c|c|c|}
\hline Method & $\mathcal{B} \rightarrow \mathcal{D}$ & $\mathcal{B} \rightarrow \mathcal{E}$ & $\mathcal{B} \rightarrow \mathcal{K}$ & $\mathcal{D} \rightarrow \mathcal{B}$ & $\mathcal{D} \rightarrow \mathcal{E}$ & $\mathcal{D} \rightarrow \mathcal{K}$ & $\mathcal{E} \rightarrow \mathcal{B}$ & $\mathcal{E} \rightarrow \mathcal{D}$ & $\mathcal{E} \rightarrow \mathcal{K}$ & $\mathcal{K} \rightarrow \mathcal{B}$ & $\mathcal{K} \rightarrow \mathcal{D}$ & $\mathcal{K} \rightarrow \mathcal{E}$ & Mean \\
\hline VFAE [4] & 9.90 & 79.20 & 81.60 & 75.50 & 78.60 & 82.20 & 72.70 & 76.50 & 85.00 & 72.00 & 73.30 & 83.80 & 78.35 \\
\hline CMD $|5|$ & .50 & 8.70 & .30 & 79.50 & 79.70 & 83.00 & 74.40 & 76.30 & 86.00 & 75.60 & 77.50 & 85.40 & 79.82 \\
\hline DANN 133 & 78.40 & 73.30 & 77.90 & 72.30 & 75.40 & 78.30 & 71.30 & 73.80 & 85.40 & 70.90 & 74.00 & 84.30 & 76.27 \\
\hline ATT 35 & 80.70 & 79.80 & 82.50 & 73.20 & 77.00 & 82.50 & 73.20 & 72.90 & 86.90 & 72.50 & 74.90 & 84.60 & 78.39 \\
\hline MT-Tr1 36] & 78.14 & 81.45 & 82.14 & 74.86 & 81.45 & 82.14 & 74.86 & 78.14 & 82.14 & 74.86 & 78.14 & 81.45 & 79.14 \\
\hline CUDA (Ours) & 82.77 & 83.07 & 85.58 & 80.02 & 82.06 & 85.70 & 75.88 & 76.05 & 87.30 & 73.08 & 73.06 & 86.66 & 80.93 \\
\hline & & & & & & & & & & & & & \\
\hline$s s=\mathcal{B} \mathcal{L}_{2}$ (Ours) & 81.07 & 75.11 & 77.53 & 77.67 & 75.99 & 79.78 & 73.12 & 74.48 & 86.19 & 72.59 & 76.24 & 85.92 & 77.97 \\
\hline$s s+$ & & & & & & & & & & & & & 78.37 \\
\hline$s s+t u+s u$ (Ours) & & & & & & & 69.70 & & & & & & 77.91 \\
\hline$s s+t u+s u+t a$ (Ours) & 82.77 & 83.07 & 85.58 & 80.02 & 82.06 & & 75.88 & & 87.30 & & & & 80.93 \\
\hline$s s+t u+s u+s a+t a$ (Ours) & 80.37 & 80.20 & 84.58 & 78.45 & 81.36 & 85.03 & 75.05 & 75.01 & 87.47 & 72.63 & 71.97 & 86.31 & 79.86 \\
\hline
\end{tabular}

guish loss (5), experimenting with better neural networks along with our contradistinguish loss (5), we observed better results in both visual and language domain adaptation task over the neural networks used in [2], [10] on visual experiments and MAN [43] on language experiments.

\section{Analysis of Experimental Results}

1) Visual Domain Adaptation: In tasks $\mathcal{C} 9 \rightarrow \mathcal{S} 9$ and $\mathcal{S S} \rightarrow \mathcal{G} \mathcal{T}, \mathcal{B} \mathcal{L}_{1}<$ CUDA in Table III $\mathcal{B L}_{1}$ is poor because $n_{t} \ll n_{s}$ causing under-fitting during only target domain supervised loss training. The improved results of CUDA indicates that CTDR is able contradistinguish on the target domain along with the transfer of informative knowledge required for the classification from a larger source domain. This indicates that CTDR is indeed successful in contradistinguishing on a relatively small set of unlabeled target domain using larger source domain information. Other interesting observation is in the task $\mathcal{C} 9 \rightarrow \mathcal{S} 9$, where $\mathcal{B} \mathcal{L}_{2}$ is slightly better than CUDA. This is due to slight over-fitting on the target domain training examples which are actually non-informative for classification leading to a small decrease in the target domain test accuracy. $\mathcal{B L}_{2}>\mathcal{B L}_{1}$ indicates source domain has more information than target domain due to large source and small target training sets. Figure $3(\mathrm{a}-\mathrm{d})$ shows t-SNE plots for $\mathcal{M N} \rightarrow \mathcal{S V}$ as the training progresses using CUDA. We indicate these plots as this is the most difficult among all the visual experiments due contrasting domains. Figure $4(a-h)$ shows t-SNE plots on the test sample outputs of CTDR for all eight visual experiments and they show clear class-wise clustering on both source and target domains indicating the efficacy of CUDA.

2) Language Domain Adaptation: In task $\mathcal{K} \rightarrow \mathcal{D}$, $\mathcal{B} \mathcal{L}_{2}>$ CUDA because of slight over-fitting on source domain. Figure $5(\mathrm{a}-\mathrm{d})$ show the t-SNE plots of top four language tasks indicating classes being oriented on either half of the line like clustering.

\section{Conclusion}

In this paper, we have proposed a simple and direct approach that addresses the problem of unsupervised domain adaptation that is different from the standard distribution alignment approaches. In our approach, we jointly learn a Contradistinguisher (CTDR) on the source and target domain distribution in the same input feature space using contradistinguish loss for unsupervised target domain to identify contrastive features. We have shown that the contrastive learning 
overcomes the need and drawbacks of domain alignment, especially in tasks where domain shift is very high (e.g., language domains) and data augmentation techniques cannot be applied. Due to the inclusion of prior enforcing in the contradistinguish loss, the proposed unsupervised domain adaptation method CUDA could incorporate any known target domain prior to overcome the drawbacks of skewness in the target domain, thereby resulting in a skew-robust model. We demonstrated the effectiveness of our model by achieving state-of-the-art results on all the visual domain adaptation tasks over eight different benchmark visual datasets and nine language domain adaptation tasks out of twelve along with the best mean test accuracy of all the twelve tasks on benchmark Amazon customer reviews sentiment analysis dataset. Specifically, the results in language domains reinforced the efficacy of CUDA on being robust to high sparsity or high domain shift tasks that pose challenges to standard domain alignment approaches.

\section{ACKNOWLEDGMENT}

The authors would like to thank Ministry of Human Resource Development (MHRD), Government of India, for their generous funding towards this work through UAY Project: IISc 001 and IISc 010.

\section{REFERENCES}

[1] P. Häusser, T. Frerix, A. Mordvintsev, and D. Cremers, "Associative domain adaptation," in ICCV, 2017.

[2] K. Saito, K. Watanabe, Y. Ushiku, and T. Harada, "Maximum classifier discrepancy for unsupervised domain adaptation," in CVPR, 2018.

[3] G. French, M. Mackiewicz, and M. Fisher, "Self-ensembling for visual domain adaptation," in ICLR, 2018.

[4] C. Louizos, K. Swersky, Y. Li, M. Welling, and R. S. Zemel, "The variational fair autoencoder," in ICLR, 2016.

[5] W. Zellinger, T. Grubinger, E. Lughofer, T. Natschläger, and S. Saminger-Platz, "Central moment discrepancy (CMD) for domain-invariant representation learning," in ICLR, 2017.

[6] M. Ghifary, W. B. Kleijn, M. Zhang, D. Balduzzi, and W. Li, "Deep reconstruction-classification networks for unsupervised domain adaptation," in $E C C V, 2016$.

[7] K. Bousmalis, G. Trigeorgis, N. Silberman, D. Krishnan, and D. Erhan, "Domain separation networks," in NIPS, 2016.

[8] Y. Ganin and V. Lempitsky, "Unsupervised domain adaptation by backpropagation," in ICML, 2015.

[9] M. Liu and O. Tuzel, "Coupled generative adversarial networks," in NIPS, 2016.

[10] E. Tzeng, J. Hoffman, K. Saenko, and T. Darrell, "Adversarial discriminative domain adaptation," in CVPR, 2017.

[11] S. Sankaranarayanan, Y. Balaji, C. D. Castillo, and R. Chellappa, "Generate to adapt: Aligning domains using generative adversarial networks," in CVPR, 2018.

[12] Y. C. Liu, Y. Y. Yeh, T. C. Fu, S. D. Wang, W. C. Chiu, and Y. C. F. Wang, "Detach and adapt: Learning cross-domain disentangled deep representation," in CVPR, 2018.

[13] P. Russo, F. M. Carlucci, T. Tommasi, and B. Caputo, "From source to target and back: Symmetric bi-directional adaptive gan," in $C V P R, 2018$.

[14] J. Hoffman, E. Tzeng, T. Park, J. Zhu, P. Isola, K. Saenko, A. Efros, and T. Darrell, "CyCADA: Cycle-consistent adversarial domain adaptation," in ICML, 2018.

[15] S. Xie, Z. Zheng, L. Chen, and C. Chen, "Learning semantic representations for unsupervised domain adaptation," in ICML, 2018.
[16] M. Long, Z. Cao, J. Wang, and M. I. Jordan, "Conditional adversarial domain adaptation," in NIPS, 2018.

[17] C. Chen, Z. Chen, B. Jiang, and X. Jin, "Joint domain alignment and discriminative feature learning for unsupervised deep domain adaptation," in $A A A I, 2019$.

[18] R. Shu, H. Bui, H. Narui, and S. Ermon, "A DIRT-t approach to unsupervised domain adaptation," in ICLR, 2018.

[19] E. Hosseini-Asl, Y. Zhou, C. Xiong, and R. Socher, "Augmented cyclic adversarial learning for low resource domain adaptation," in ICLR, 2019.

[20] V. N. Vapnik, "An overview of statistical learning theory," IEEE transactions on neural networks, 1999.

[21] G. Pandey and A. Dukkipati, "Unsupervised feature learning with discriminative encoder," in ICDM, 2017.

[22] Y. LeCun, B. Boser, J. S. Denker, D. Henderson, R. E. Howard, W. Hubbard, and L. D. Jackel, "Backpropagation applied to handwritten zip code recognition," Neural Computation, 1989.

[23] Y. LeCun, L. Bottou, Y. Bengio, P. Haffner et al., "Gradientbased learning applied to document recognition," IEEE, 1998

[24] Y. Netzer, T. Wang, A. Coates, A. Bissacco, B. Wu, and A. Y. Ng, "Reading digits in natural images with unsupervised feature learning," in NIPS Workshop on Deep Learning and Unsupervised Feature Learning, 2011.

[25] A. Krizhevsky, "Learning multiple layers of features from tiny images," Citeseer, Tech. Rep., 2009.

[26] A. Coates, A. Ng, and H. Lee, "An analysis of single-layer networks in unsupervised feature learning," in AISTATS, 2011.

[27] J. Stallkamp, M. Schlipsing, J. Salmen, and C. Igel, "The German traffic sign recognition benchmark: A multi-class classification competition," in IJCNN, 2011.

[28] J. Blitzer, R. McDonald, and F. Pereira, "Domain adaptation with structural correspondence learning," in EMNLP, 2006.

[29] A. Gretton, K. Fukumizu, Z. Harchaoui, and B. K. Sriperumbudur, "A fast, consistent kernel two-sample test," in NIPS, 2009.

[30] A. Tarvainen and H. Valpola, "Mean teachers are better role models: Weight-averaged consistency targets improve semisupervised deep learning results," in NIPS, 2017.

[31] S. Laine and T. Aila, "Temporal ensembling for semisupervised learning," in ICLR, 2017.

[32] D. P. Kingma and M. Welling, "Auto-encoding variational bayes," in ICLR, 2014

[33] Y. Ganin, E. Ustinova, H. Ajakan, P. Germain, H. Larochelle, F. Laviolette, M. Marchand, and V. Lempitsky, "Domainadversarial training of neural networks," JMLR, 2016.

[34] I. J. Goodfellow, J. Pouget-Abadie, M. Mirza, B. Xu, D. Warde-Farley, S. Ozair, A. Courville, and Y. Bengio, "Generative adversarial nets," in NIPS, 2014.

[35] K. Saito, Y. Ushiku, and T. Harada, "Asymmetric tri-training for unsupervised domain adaptation," in ICML, 2017.

[36] S. Ruder and B. Plank, "Strong baselines for neural semisupervised learning under domain shift," in $A C L, 2018$.

[37] D.-H. Lee, "Pseudo-label: The simple and efficient semisupervised learning method for deep neural networks," ICML Workshop (WREPL), 2013.

[38] Y. Grandvalet and Y. Bengio, "Semi-supervised learning by entropy minimization," in NIPS, 2005.

[39] C. L. Li, W. C. Chang, Y. Cheng, Y. Yang, and B. Póczos, "MMD GAN: towards deeper understanding of moment matching network," in NIPS, 2017.

[40] L. van der Maaten and G. Hinton, "Visualizing data using t-SNE," JMLR, 2008.

[41] M. Chen, Z. Xu, K. Q. Weinberger, and F. Sha, "Marginalized denoising autoencoders for domain adaptation," in ICML, 2012.

[42] A. Paszke, S. Gross, S. Chintala, G. Chanan, E. Yang, Z. DeVito, Z. Lin, A. Desmaison, L. Antiga, and A. Lerer, "Automatic differentiation in pytorch," NIPS Workshops, 2017.

[43] X. Chen and C. Cardie, "Multinomial adversarial networks for multi-domain text classification," in NAACL-HLT, 2018. 